\title{
Bioassay-guided isolation of a novel chemoattractant for Ralstonia solanacearum in tomato root exudates
}

\author{
Takuya Hasegawa ${ }^{1}$ - Atsushi Okabe ${ }^{2} \cdot$ Yusuke Kato $^{2} \cdot$ Atsushi Ooshiro $^{3} \cdot$ \\ Hiroshi Kawaide ${ }^{4} \cdot$ Masahiro Natsume $^{4}(\mathbb{C}$
}

Received: 25 April 2017 / Accepted: 18 August 2017 / Published online: 2 November 2017

(C) The Author(s) 2017. This article is an open access publication

\begin{abstract}
Tomato root exudates were analyzed using a bioassay to detect the chemoattractant for Ralstonia solanacearum. An activated charcoal-adsorbed fraction of root exudates from tomato cultivar Oogata-fukuju had chemoattractant activity for $R$. solanacearum strain MAFF 730138. The active component, purified using a Sep-Pak C18 cartridge, an activated charcoal column, diol-modified silica gel, and $\mathrm{NH}_{2}$-modified silica gel, is a new hydrophobic attractant. The final purified fraction produced a single peak in a diol-modified silica gel HPLC analysis.
\end{abstract}

Keywords Ralstonia solanacearum - Bacterial wilt . Chemotaxis $\cdot$ Tomato $\cdot$ Root exudates

Takuya Hasegawa and Atsushi Okabe contribute equally to this work.

Masahiro Natsume

natsume@cc.tuat.ac.jp

1 Department of Biological Production Science, United Graduate School of Agricultural Science, Tokyo University of Agriculture and Technology, Saiwai-cho, Fuchu, Tokyo 183-8509, Japan

2 Department of Bioregulation and Biointeraction, Graduate School of Agriculture, Tokyo University of Agriculture and Technology, Saiwai-cho, Fuchu, Tokyo 183-8509, Japan

3 Okinawa Prefectural Agricultural Research Center, Makabe, Itoman, Okinawa 901-0336, Japan

4 Division of Bioregulation and Biointeraction, Institute of Agriculture, Tokyo University of Agriculture and Technology, Saiwai-cho, Fuchu, Tokyo 183-8509, Japan

\section{Introduction}

Bacterial wilt caused by Ralstonia solanacearum (Yabuuchi et al. 1995) is one of the most devastating bacterial plant diseases in tropical, subtropical, and warm temperate regions worldwide (Hayward 1991). Symptoms include chlorosis, stunted growth, and wilting. Susceptible plants usually die soon after being infected. $R$. solanacearum is a soil-borne Gram-negative bacterium that infects more than 200 plant species from 50 families, including economically important crops such as tomato, potato, eggplant, pepper, tobacco and banana (Elphinstone 2005). This bacterial plant pathogen can survive long periods in water, soil, and latently infected plants (Álvarez et al. 2008). It can be transmitted via soil, water, farm equipment, and infected plant materials. Because of its wide geographic distribution, extensive host range, and tolerance to various environmental conditions, $R$. solanacearum is one of the most destructive crop pathogens worldwide.

Many factors contribute to bacterial wilt disease, including extracellular polysaccharides, enzymes that degrade plant cell walls, and type-III-secreted effector proteins (Genin and Denny 2012). Chemotaxis is another important factor influencing virulence (Kang et al. 2002; Tans-Kersten et al. 2001).

Chemotaxis, which refers to the movement of an organism toward or away from a chemical agent, is an important factor influencing microbe-plant interactions (e.g., infection, symbiosis, and root colonization). As part of the symbiotic relationship between rhizobia and leguminous plants, Rhizobium species detect a specific flavonoid released by the host plant and migrate toward the plant roots (Aguilar et al. 1988; Yoneyama and Natsume 2010). Rhizobium meliloti exhibits positive chemotaxis specifically toward the flavone luteorin, but not toward 
the related flavonoids naringenin and apigenin. Luteolin upregulates nod $A B C$ gene expression, which induces cortical cell division and root hair curling in the host plant, during the initial stages of the symbiotic relationship (Caetano-Anollés et al. 1988). The pathogenicity of Agrobacterium tumefaciens, which is responsible for the development of crown gall tumors in susceptible plants, is also affected by chemotactic activities (Hawes and Smith 1989). Chemotaxis also mediates the colonization of root surfaces by plant-growth-promoting rhizobacteria and biocontrol agents, including Bacillus and Pseudomonas spp. (de Weert et al. 2002; Oku et al. 2014; Zhang et al. 2014).

In early studies on rhizobia, researchers attempted to fractionate root exudates and identify chemoattractants. A study on Rhizobium leguminosarum and pea (Pisum sativum L.) root exudates revealed a fraction that contained substances with molecular weights $<1000$ and a cationic fraction produced stronger chemotactic responses than neutral or anionic fractions (Gaworzewska and Carlile 1982). The amino acids in the cationic fraction, sugars in the neutral fraction, and organic acids in the anionic fraction were analyzed, as were the associated chemotactic activities. Another study examined the influence of chemotaxis on the interactions between Bradyrhizobium japonicum and soybean seed and root exudates. Most of the chemotactic activity was detected in the flow-through fraction collected from a Sep-Pak C18 cartridge. The major attractants were identified as dicarboxylic acids, including succinate and malonate, as well as the amino acids glutamic acid and aspartic acid (Barbour et al. 1991).

In $R$. solanacearum, an immotile af lagellate mutant was found to be significantly less virulent than the wildtype bacterium (Tans-Kersten et al. 2001). Additionally, root exudates from tomato (i.e., a host plant) produced stronger positive chemotactic activity than root exudates from rice (i.e., a nonhost plant). The chemoattractants in the tomato root exudates were determined to be heat stable and unaffected by proteinase K (Yao and Allen 2006). A subsequent analysis of the amino acids, organic acids, and sugars indicated that several amino acids, including aspartic acid, proline, and glutamine, are potentially strong chemoattractants. However the exact components of the tomato root exudates affecting the chemotaxis of $R$. solanacearum have not been fully characterized.

We hypothesized that the specific substance in host plant root exudates that attracts pathogens is not a common substance such as amino acids, organic acids or sugars. The objective of this study was to identify the substance(s) in tomato root exudates that are attractive to $R$. solanacearum using a bioassay-guided approach.

\section{Materials and methods}

\section{Bacterial strains}

Seven race 1 strains of $R$. solanacearum that had been isolated from tomato were obtained from The Genetic Resources Center, National Agriculture and Food Research Organization, Japan (Table 1). These strains were screened to identify an appropriate bioassay strain. Strain MAFF730138, which showed positive chemotaxis toward the chemoattractant released by tomato cv. Oogata-fukuju, was used in the bioassay-guided isolation of the chemoattractant.

\section{Collection of tomato root exudates}

Five tomato (Solanum lycopersicum L.) cultivars were used in these experiments: Momotaro, Ponderosa, Sekai-ichi, Oogata-fukuju, and Chika. For each of the five cultivars, seeds were surface-sterilized for $6 \mathrm{~min}$ in an aqueous solution of $0.8 \% \mathrm{w} / \mathrm{v}$ sodium hypochlorite and $0.1 \% \mathrm{v} / \mathrm{v}$ Tween 80 . The seeds were then washed twice in sterile water. Fifty seeds were transferred to a 1-L beaker lined with gauze, and then moistened with $10 \mathrm{~mL}$ sterile deionized water. The beaker was sealed with plastic film and an elastic band, and then incubated in the dark at $28{ }^{\circ} \mathrm{C}$ for 3 days. After the seeds germinated, the water in the beaker was collected and replaced with $20 \mathrm{~mL}$ fresh sterile deionized water for further incubation for 1 week in a growth chamber with $12 \mathrm{~h}$ light $\left(25^{\circ} \mathrm{C}\right) / 12 \mathrm{~h}$ dark $\left(15^{\circ} \mathrm{C}\right)$ cycle. On day 3 of incubation, the water in the beaker was collected and replaced with $20 \mathrm{~mL}$ fresh sterile deionized water. After 7 days, the water in the beaker was collected and combined with the previously collected water to constitute the root exudate (approximately $50 \mathrm{~mL}$ ). The new chemoattractant was isolated from the tomato cv. Oogata-fukuju.

\section{Fractionation of root exudates}

Root exudates were applied to an activated charcoal column. The column was washed with water, after which the adsorbed substances were eluted with $80 \%$ v/v aqueous

Table 1 Strains of Ralstonia solanacearum used in this study

\begin{tabular}{lllll}
\hline MAFF no. & Race & Biovar & Collection site & Year isolated \\
\hline 211514 & 1 & 3 & Kochi & 1990 \\
301070 & 1 & 3 & Kochi & 1966 \\
301485 & 1 & N2 & Oita & 1984 \\
730102 & 1 & 4 & Nara & 1982 \\
730128 & 1 & N2 & Mie & 1982 \\
730138 & 1 & 3 & Kochi & 1983 \\
730141 & 1 & 4 & Shiga & 1984 \\
\hline
\end{tabular}


acetone. The flow-through and wash fractions were combined and lyophilized to obtain the water-soluble fraction. The aqueous acetone eluate was concentrated to dryness in vacuo to obtain the activated charcoal-adsorbed fraction.

\section{Chemotaxis assay}

The semisolid agar plate method developed by Okon et al. (1980) was modified as described by Yao and Allen (2006). $R$. solanacearum was grown overnight in CPG medium (Hendrick and Sequeira 1984) at $28{ }^{\circ} \mathrm{C}$ on a rotary shaker (90 rpm). The cultures were centrifuged at $4700 \times g$ for $10 \mathrm{~min}$, and the pelleted bacteria were washed twice with sterile chemotaxis buffer (Yao and Allen 2006). The bacteria were then resuspended in the same buffer to an $\mathrm{OD}_{600}$ of 2.0. A $20-\mu \mathrm{L}$ aliquot of the bacterial suspension was placed onto the center of a Petri dish (6 cm i.d.) containing $2.8 \mathrm{~mL}$ semisolid medium (i.e., chemotaxis buffer solidified with $0.3 \%$ agar). A sample-containing paper disc ( $8 \mathrm{~mm}$ diameter, $1.5 \mathrm{~mm}$ thick) was placed on the medium $20 \mathrm{~mm}$ from the edge of the bacterial suspension. A paper disc containing chemotaxis buffer was placed at the opposite position of the sample disc as a control. Glutamine-containing discs ( 2 and $0.2 \mu \mathrm{mol} /$ disc) served as positive controls. Medium containing the bacterial suspension but with no paper disc was used as a negative control. The plates were incubated at $28{ }^{\circ} \mathrm{C}$ for 7 days. The distance migrated by the bacterial suspension was measured at 3 and 7 days after the start of the experiment. When selecting the bioassay strain, the relationship between the migration distance and incubation period was visualized with line graphs (Figs. 1, 2). During the purification of the chemoattractant, the value obtained by subtracting the distance migrated toward the control disc from the distance migrated toward the sample disc at day 7 was used as the index of chemotactic activity (Fig. 4). All assays were repeated at least three times.

\section{Results}

\section{Selection of $\boldsymbol{R}$. solanacearum strain for bioassay}

Seven $R$. solanacearum strains were screened to identify a suitable strain for the chemotaxis assay. The strains were first screened according to their response to glutamine (2 and $0.2 \mu \mathrm{mol} / \mathrm{disc}$ ). Although the strains showed different migration speed and sensitivity, all of them exhibited positive chemotaxis toward glutamine (Fig. 1). All strains had reached the glutamine-containing paper discs by 7 days after the start of the incubation period. Analyses on day 3 revealed that strain MAFF 730102 exhibited the strongest response to $0.2 \mu \mathrm{mol}$ glutamine, followed by MAFF 211514 , and 730138. There were no significant differences between the distance migrated toward the disc containing $0.2 \mu \mathrm{mol}$ of glutamine and the distance migrated toward the control disc for strains MAFF 301070, 730128, and 730141. Because the bacterial halo was unclear for strains MAFF 301070 and 730128 , these two strains were eliminated as bioassay strain candidates.

The chemotactic responses of the five remaining $R$. solanacearum strains were examined with crude extracts of tomato root exudates. The root exudate of the tomato cultivar Momotaro was divided into fractions that adsorbed to activated charcoal and that were water-soluble. The fractions were then used at an amount equivalent to 10 seedlings. All five $R$. solanacearum strains exhibited a chemotactic
Fig. 1 Chemotactic response of Ralstonia solanacearum strains to glutamine. Filled square, empty square: $2 \mu \mathrm{mol}$ glutamine and control; filled circle, empty circle: $0.2 \mu \mathrm{mol}$ glutamine and control; empty diamond: negative control. Error bars indicate standard errors $(n=3)$

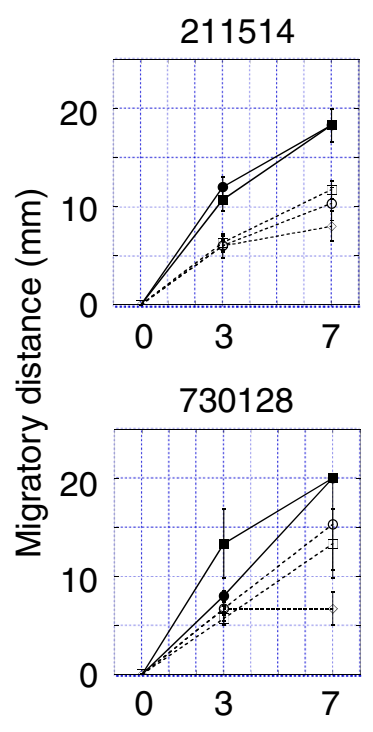

301070

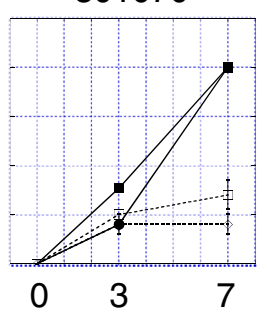

730138

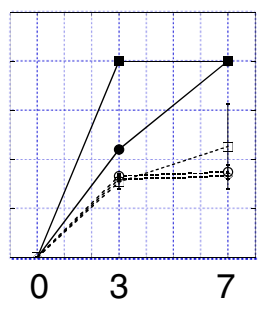

Incubation period (days)
301485

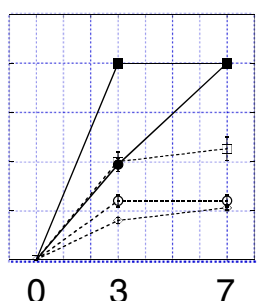

730141

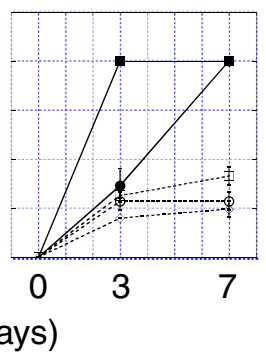

730102

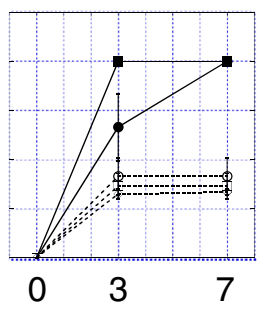


Fig. 2 Tomato root exudate chemoattractant activity for Ralstonia solanacearum MAFF730138. Filled square, empty square activated charcoal-adsorbed fraction and control; filled circle, empty circle water-soluble fraction and control; empty diamond negative control. Error bars indicate standard errors $(n=3)$

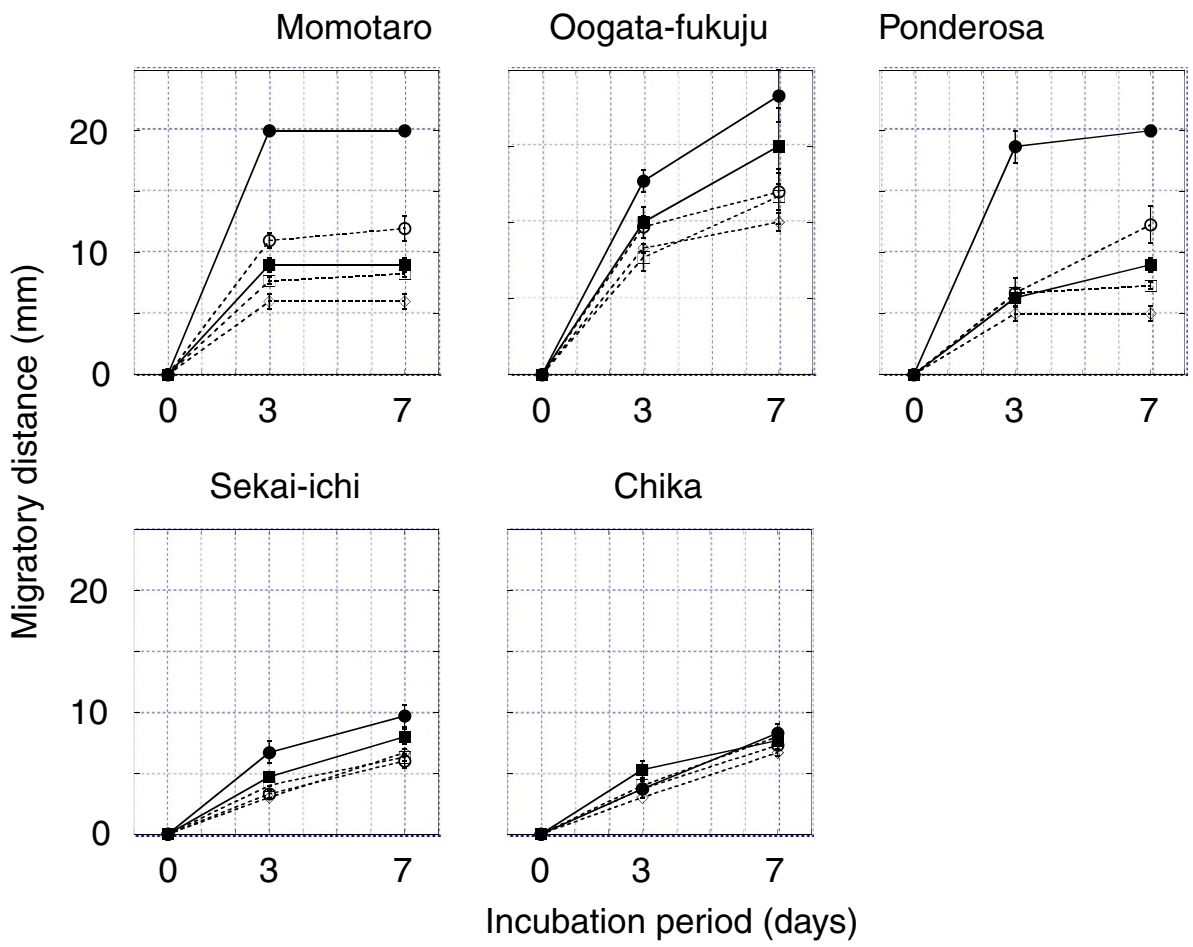

response only to the water-soluble fraction (data not shown). Strains MAFF 211514, 730138 and 730141 showed similar responses to the water-soluble fraction and glutamine ( $2 \mu \mathrm{mol} / \mathrm{disc}$ ), while the other two strains had weaker responses to the water-soluble fraction than to glutamine. Thus, these three strains were used in further analyses.

\section{Selection of tomato cultivar for producing the chemoattractant}

Root exudates were prepared from tomato cultivars Momotaro, Ponderosa, Sekai-ichi, Oogata-fukuju and Chika (cherry tomato). The total root exudate yield (i.e., sum of activated charcoal-adsorbed and water-soluble fractions) from 100 seedlings was approximately $7 \mathrm{mg}$ for Chika and $12 \mathrm{mg}$ each for the other four tomato cultivars. The chemoattractant activities of the tomato root exudates were first tested using $R$. solanacearum MAFF 730138. The water-soluble fractions of Momotaro and Ponderosa exhibited strong activities, followed by Oogata-fukuju (Fig. 2). Among the activated charcoal-adsorbed fractions, only that from Oogata-fukuju exhibited chemoattractant activity, although it was not statistically significant in this experiment. None of the fractions from Sekai-ichi and Chika showed chemoattractant activity.

We therefore prepared the activated charcoal-adsorbed fraction of Oogata-fukuju again and examined its chemoattractant activity using three strains selected in the experiment described in the previous section. As a result, $R$. solanacearum MAFF 730138 reproducibly exhibited significant response to the fraction, and strains MAFF 211514 and MAFF 730141 showed weak responses (data not shown). These results led us to speculate that Oogatafukuju produces a previously unreported hydrophobic chemoattractant and prompted us to attempt to purify it, using $R$. solanacearum MAFF 730138 as the bioassay strain.

\section{Isolation of the chemoattractant from the activated charcoal-adsorbed fraction of Oogata-fukuju root exudate}

The suitability of several purification methods was examined. The activated charcoal-adsorbed fraction was divided into four parts, which were extracted with ethyl acetate or fractionated with a Sep-Pak C18 cartridge (Nihon Waters, Tokyo, Japan), an Oasis HLB cartridge (Nihon Waters) or Chromatorex-DIOL (diol-modified silica gel; Fuji Silysia Chemical, Kasugai, Aichi, Japan). Chemotactic activities were observed in the water phase of the ethyl acetate extraction and in the flow-through fractions collected from the Sep-Pak C18 and Oasis HLB cartridges. Therefore, the root exudates were purified using a Sep-Pak C18 cartridge before being applied to the activated charcoal column for subsequent purification of the substance. During the chromatographic separation using diol-silica gel, chemoattractant activities were observed in the $10 \% \mathrm{MeOH}-\mathrm{CHCl}_{3}$ fraction. 


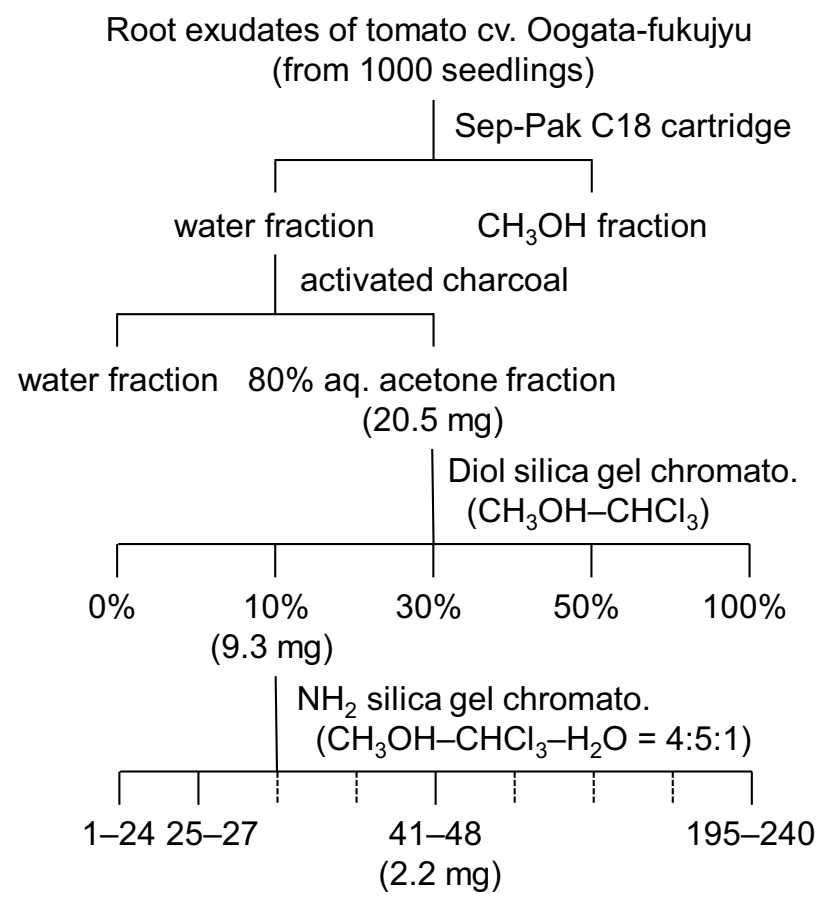

Fig. 3 Isolation protocol for chemoattractant from root exudate of tomato cultivar Oogata-fukuju. aq. aqueous, chromato. chromatography

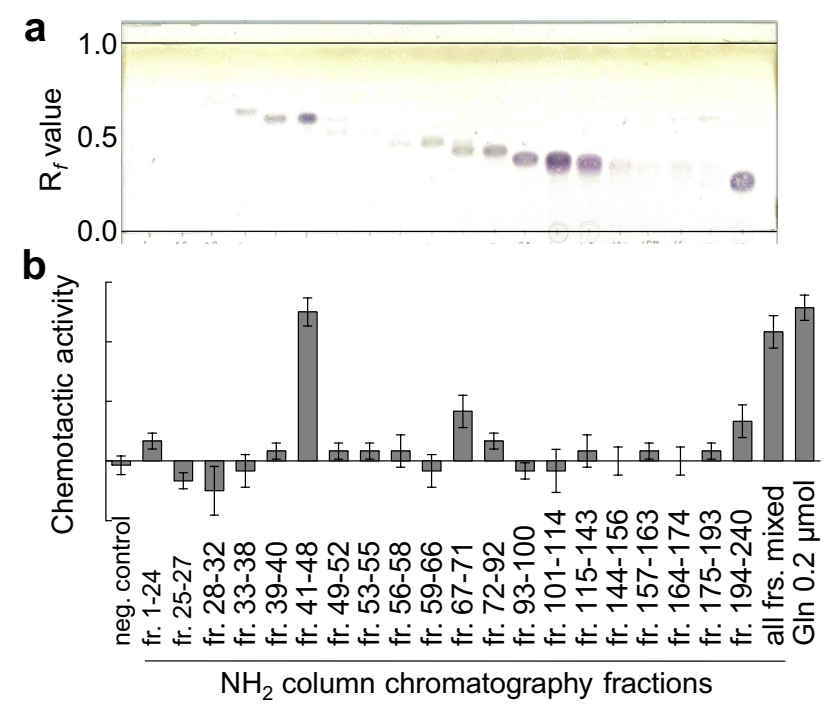

Fig. 4 Thin layer chromatography analysis (a) and chemotactic activity (b) of $\mathrm{NH}_{2}$-modified silica gel column chromatography fractions. A silica gel plate, $\mathrm{CH}_{3} \mathrm{OH}-\mathrm{CHCl}_{3}-\mathrm{H}_{2} \mathrm{O}$ (10:15:2) solvent and $\alpha$-naphthol-sulfuric acid visualization reagent were used. Samples of each fraction equivalent to exudates from 50 seedlings were used for the bioassay. Error bars indicate standard errors $(n=3)$

The active fraction collected from the diol-silica gel column was subjected to thin layer chromatography (TLC) and then its components were visualized by spraying with various reagents. The active fraction reacted with the

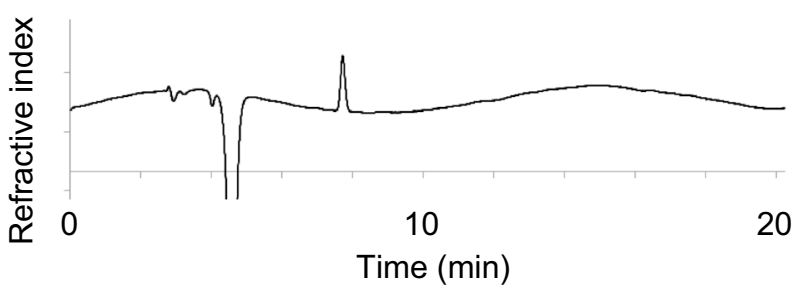

Fig. 5 HPLC chromatogram of isolated chemoattractant. An Inertsil Diol $(5 \mu \mathrm{m}, 4.6 \times 250 \mathrm{~mm})$ column with a mobile phase of $\mathrm{CH}_{3} \mathrm{CN}-$ $\mathrm{H}_{2} \mathrm{O}$ (95:5) and flow rate of $1 \mathrm{~mL} / \mathrm{min}$ were used with refractive index detection

$\alpha$-naphthol-sulfuric acid reagent (for sugars), but not with dinitrophenylhydrazine (for aldehyde and keto groups), ninhydrin (for amino acids, amines and amino-sugars), or Dragendorff (for nitrogen-containing compounds) reagents.

Because the active substance was likely to be a sugar-containing compound, Wakosil 50NH2(HC) (i.e., $\mathrm{NH}_{2}$-modified silica gel; Wako Pure Chemical Industries, Osaka, Japan) was used for the subsequent purification step (Fig. 3). The eluate was collected from the column with a fraction collector and then analyzed by TLC. Fractions producing a spot with the same $\mathrm{R}_{f}$ value and color reaction were combined (in total, 20 fractions; Figs. 3, 4a). Fractions 41-48 had strong chemoattractant activity, and fractions 67-71 had weak activity (Fig. 4b). In this assay, the dose of fractions $41-48$ was $120 \mu \mathrm{g} / \mathrm{disc}$, and the resulting activity was comparable to that of $0.2 \mu \mathrm{mol}$ glutamine $(30 \mu \mathrm{g} / \mathrm{disc})$. The HPLC analysis revealed that fractions $41-48$ produced a single peak (Fig. 5), suggesting that the chemoattractant had been isolated. A comparison of the chemoattractant with authentic sugars on a TLC plate (Fig. 6a) revealed that the chemoattractant had a higher $\mathrm{R}_{f}$ value than those of mono-, di-, and trisaccharides and obviously differed from the sugars commonly found in plant root exudates. Of the analyzed sugars, galactose had very weak chemoattractant activity at $4 \mu \mathrm{mol}(720 \mu \mathrm{g} / \mathrm{disc})$, while the others had no chemoattractant activity (Fig. 6b).

\section{Discussion}

Most other chemotaxis studies focused only on substances known to be present in plant root exudates, including amino acids, organic acids, and sugars. However, these compounds can be excreted by many kinds of plants and would not be specific to a host plant. Yao and Allen (2006) reported that $R$. solanacearum was more strongly attracted to tomato (a host plant) root exudates than to rice (a nonhost plant) root exudates. However, the chemoattractant(s) in tomato root exudates had not been identified. We hypothesized that $R$. solanacearum pathogenic to tomato must respond to specific 
Fig. 6 TLC analysis (a) and chemoattractant activity (b) of isolated chemoattractant and authentic sugars. Silica gel plate, $\mathrm{CH}_{3} \mathrm{OH}-\mathrm{CHCl}_{3}-$ $\mathrm{H}_{2} \mathrm{O}(10: 15: 2)$ solvent and $\alpha$-naphthol-sulfuric acid visualization reagent were used. Chemoattractant activity was scored using a relative scale of 0 (none) to 3 (high) a

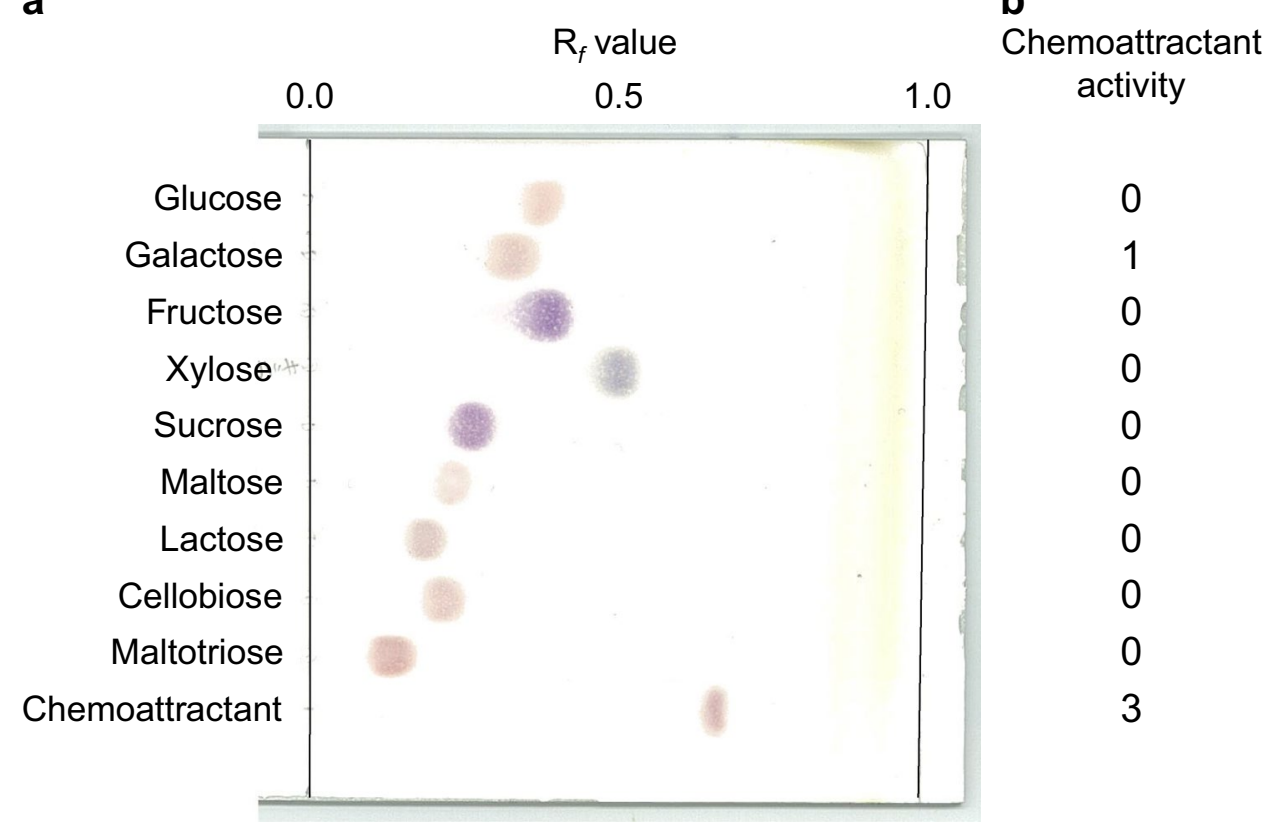

chemoattractant(s) produced by tomato, so we attempted to isolate the substance by a bioassay-guided fractionation procedure. To the best of our knowledge, this is the first report of an attempt to identify chemoattractants in root exudates based on activity.

A relationship between chemotaxis and virulence has been reported previously (e.g., Tans-Kersten et al. 2001), but it is unknown whether a strain's virulence is related to its chemotactic ability. It is also unknown whether the susceptibility of a given tomato cultivar is related to the chemoattractant activity of its root exudates. To explore the chemoattractant activity of tomato root exudate, we selected from five tomato cultivars a tomato cultivar that produces a chemoattractant and from seven $R$. solanacearum strains a strain that responds to the chemoattractant. For the bioassay, we selected race 1 strain MAFF 730138 of $R$. solanacearum because it is known to infect tomato and had chemotactic activity in the screening test, and we selected tomato cultivar Oogata-fukuju because its root exudate had chemoattractant activity in the screening test. Tomato is one of the most cultivated and improved crops. In addition, Oogatafukuju was developed in 1952, so it is relatively old compared with those currently available, and these older varieties are generally susceptible to bacterial wilt. Considering that one of the objectives of crop breeding is to enhance disease resistance, it is interesting that among the varieties tested, the older variety Oogata-fukuju was found to produce a chemoattractant.

The chemoattractant we found was moderately hydrophobic; it passed through a Sep-Pak C18 cartridge and adsorbed to activated carbon. It also reacted to the coloring reagent for sugar. These results indicate that the active substance is a glycoside or some type of sugar derivative.

Detailed studies on the structure, biological activities and specificity of the chemoattractant are now underway. We are also examining the relationship between susceptibility of tomato cultivars and the chemoattractant activity of their root exudates, which might inform breeding of improved tomato varieties.

Acknowledgements The authors thank Dr. Kazuhiro Nakaho (National Agriculture and Food Research Organization) for technical advice on handling $R$. solanacearum. This work was supported by JSPS KAKENHI grants JP23580146, JP26450137 and JP17H03812.

Author contributions The study was conceived by AOo and MN. YK established the bioassay method. AOk contributed to the selection of the $R$. solanacearum bioassay strain and the tomato cultivar. TH purified the chemoattractants from the tomato cultivar using the bioassay strain. HK contributed to the experimental design and data analysis. The manuscript was written by $\mathrm{TH}$ and $\mathrm{MN}$ and reviewed by all authors.

Open Access This article is distributed under the terms of the Creative Commons Attribution 4.0 International License (http://creativecommons.org/licenses/by/4.0/), which permits unrestricted use, distribution, and reproduction in any medium, provided you give appropriate credit to the original author(s) and the source, provide a link to the Creative Commons license, and indicate if changes were made.

\section{References}

Aguilar JMM, Ashby AM, Richards AJM, Loake GJ, Watson MD, Shaw CH (1988) Chemotaxis of Rhizobium leguminosarum biovar 
phaseoli towards flavonoid inducers of the symbiotic nodulation genes. J Gen Microbiol 134:2741-2746

Álvarez B, López MM, Biosca EG (2008) Survival strategies and pathogenicity of Ralstonia solanacearum phylotype II subjected to prolonged starvation in environmental water microcosms. Microbiology 154:3590-3598

Barbour WM, Hattermann DR, Stacey G (1991) Chemotaxis of Bradyrhizobium japonicum to soybean exudates. Appl Environ Microbiol 57:2635-2639

Caetano-Anollés G, Crist-Estes DK, Bauer WD (1988) Chemotaxis of Rhizobium meliloti to the plant flavone luteorin requires functional nodulation genes. J Bacteriol 170:3164-3169

de Weert S, Vermeiren H, Mulders IHM, Kuiper I, Hendrickx N, Bloemberg GV, Vanderleyden J, De Mot R, Lugtenberg BJJ (2002) Flagella-driven chemotaxis towards exudate components is an important trait for tomato root colonization by Pseudomonas fluorescens. Mol Plant-Microbe Interact 15:1173-1180

Elphinstone JG (2005) The current bacterial wilt situation: A global view. In: Allen C, Prior P, Hayward AC (eds) Bacterial wilt disease and the Ralstonia solanacearum species complex. APS, St. Paul, pp 9-28

Gaworzewska ET, Carlile MJ (1982) Positive chemotaxis of Rhizobium leguminosarum and other bacteria towards root exudates from legumes and other plants. J Gen Microbiol 128:1179-1188

Genin S, Denny TP (2012) Pathogenomics of the Ralstonia solanacearum species complex. Annu Rev Phytopathol 50:67-89

Hawes MC, Smith LY (1989) Requirement for chemotaxis in pathogenicity of Agrobacterium tumefaciens on roots of soil-grown pea plants. J Bacteriol 171:5668-5671

Hayward AC (1991) Biology and epidemiology of bacterial wilt caused by Pseudomonas solanacearum. Annu Rev Phytopathol 29:65-87
Hendrick CA, Sequeira L (1984) Lipopolysaccharide-defective mutants of the wilt pathogen Pseudomonas solanacearum. Appl Environ Microbiol 48:94-101

Kang Y, Liu H, Genin S, Schell MA, Denny TP (2002) Ralstonia solanacearum requires type 4 pili to adhere to multiple surfaces and for natural transformation and virulence. Mol Microbiol 46:427-437

Okon Y, Cakmakci L, Nur I, Chet I (1980) Aerotaxis and chemotaxis of Azospirillum brasilense: a note. Microb Ecol 6:277-280

Oku S, Komatsu A, Nakashimada Y, Tajima T, Kato J (2014) Identification of Pseudomonas fluorescens chemotaxis sensory proteins for malate, succinate, and fumarate, and their involvement in root colonization. Microbes Environ 29:413-419

Tans-Kersten J, Huang H, Allen C (2001) Ralstonia solanacearum needs motility for invasive virulence on tomato. J Bacteriol 183:3597-3605

Yabuuchi E, Kosako Y, Yano I, Hotta H, Nishiuchi Y (1995) Transfer of two Burkholderia and an Alcaligenes species to Ralstonia gen. nov.: proposal of Ralstonia pickettii (Ralston, Palleroni and Doudoroff, 1973) comb. nov., Ralstonia solanacearum (Smith 1896) comb. nov. and Ralstonia eutropha (Davis, 1969) comb. nov. Microbiol Immunol 39:897-904

Yao J, Allen C (2006) Chemotaxis is required for virulence and competitive fitness of the bacterial wilt pathogen Ralstonia solanacearum. J Bacteriol 188:3697-3708

Yoneyama K, Natsume M (2010) Allelochemicals for plant-plant and plant-microbe interactions. In: Mori K (ed) Comprehensive natural products II. Chemistry and biology, vol 4. Elsevier, Amsterdam, pp 539-561

Zhang N, Wang D, Liu Y, Li S, Shen Q, Zhang R (2014) Effects of different plant root exudates and their organic acid components on chemotaxis, biofilm formation and colonization by beneficial rhizosphere-associated bacterial strains. Plant Soil 374:689-700 